\title{
Applications of Combined Transmission Kikuchi Diffraction and STEM-SDD X- Ray Analysis in Irradiated Materials
}

\author{
Chad M. Parish ${ }^{1}$, Kun Wang ${ }^{1}$, Philip Edmondson ${ }^{1}$, David T. Hoelzer ${ }^{1}$, Quinlan Smith ${ }^{1}$ and Yutai Katoh ${ }^{1}$ \\ 1. Oak Ridge National Laboratory, Oak Ridge, TN.
}

Individually, transmission Kikuchi diffraction (tKD) [1] and scanning transmission electron microscopy (STEM) X-ray mapping, particularly with modern 4-sector large area silicon drift detectors (SDDs), are powerful techniques for materials analysis. Combined, however, tKD and STEM-X ray mapping provide tremendous correlative data for interpretation of material properties in terms of microstructures [2]. This is particularly important for nuclear materials, where combinations of high temperature, corrosive environments, and punishing radiation fields (and atomic transmutations) drive radical microstructural changes and degradation of mechanical and functional properties.

As an example, Fig. 1 shows combined tKD and X-ray analysis from a nanostructured ferritic alloy (NFA) 14YWT, in which grain boundary crystallographic character is measured via tKD and grain boundary segregation is measured via STEM. The specimen in Figure 1 has been subjected to ion irradiation to high dose at cryogenic temperature [3], and an SEM-forward scatter detector (FSD) image (Fig 1a) is shown, with visible grains. tKD data was acquired (Versa3D DualBeam with Oxford Instruments Nordlys EBSD camera and Oxford Instrument tKD holder) and is presented in Figs. 1b-1c. In Fig. 1b, the pattern quality is mapped, with overlaid grain boundaries colored (see inset) from $2^{\circ}$ to $65^{\circ}$ misorientation. This is vital information, because radiation damage drives grain boundary processes with a highly non-monotonic dependence on grain boundary misorientation [e.g., 4]. The Z-projected inverse pole figure coloration is shown in Fig 1c, confirming the very fine grain structure is measurable via tKD. This same sample region is suitable for examination using advanced STEM methods (Talos F200X "ChemiSTEM"). An advanced 4-sector SDD and high-brightness probe achieves large-area mapping at $1024^{2}$ pixels, Figs. 1d-1g. Small TiN precipitates are visible in Fig. 1f: they are sharply defined deep in the material and blurred in the irradiated (shallow) region due to ballistic mixing [3]. Ti, $\mathrm{Cr}$, and $\mathrm{W}$ segregation to grain boundaries is visible in the deeper areas, and expected to be a strong function of grain boundary orientation. These combined datasets illustrate the ability of tKD and SDDbased STEM mapping to provide comprehensive characterization of material states.

Similarly, neutron-irradiated tungsten is shown in Fig. 2 (taken with the same instrumentation as Fig. 1). An FSD image (Fig 2a) shows fine grains, and tKD data is given in Fig. 2b; the matrix is BCC, and the triangular precipitate in the center is HCP. STEM-BF (Fig. 2c) and X-ray maps for transmutant atoms (Re, Os) bred during irradiation (Fig. 2d) indicate massive intragranular precipitation, a large triple-point precipitate, and copious grain boundary radiation-induced segregation. This illustrates how the combination of tKD and SDD-based X-ray spectrometry allows large numbers of grains and grain boundaries to be comprehensively characterized with high efficiency ( $\sim 1$ hour each for tKD and STEM maps). Overall, the combination of tKD and STEM-SDD mapping provides a new and as-yet underutilized approach to correlative materials characterization [5].

References:

[1] GC Sneddon et al, Mat. Sci. Eng. R., 110 (2019), p. 1.

[2] CM Parish, K Wang, and PD Edmondson, Scripta Mater. 143 (2018), p. 169. 
[3] CM Parish et al, J. Nucl. Mater. 445 (2014), p. 251.

[4] KG Field et al, J. Nucl. Mater. 435 (2013), p. 172.

[5] 14YWT work supported by US Department of Energy, Office of Science, Basic Energy Sciences, Materials Science and Engineering Division; instrument access was supported by the US Department of Energy, Office of Nuclear Energy under DOE Idaho Operations Office Contrast DE-AC07-051D14517 as part of a Nuclear Science User Facilities experiment. Tungsten work supported by an Early Career Award, US Department of Energy, Office of Science, Fusion Energy Sciences, under contract number DE-AC05-00OR22725. Talos F200X instrument supported by the Department of Energy, Office of Nuclear Energy, Fuel Cycle R\&D Program and the Nuclear Science User Facilities. A portion of this research used resources at the High Flux Isotope Reactor, a DOE Office of Science User Facility operated by the Oak Ridge National Laboratory.
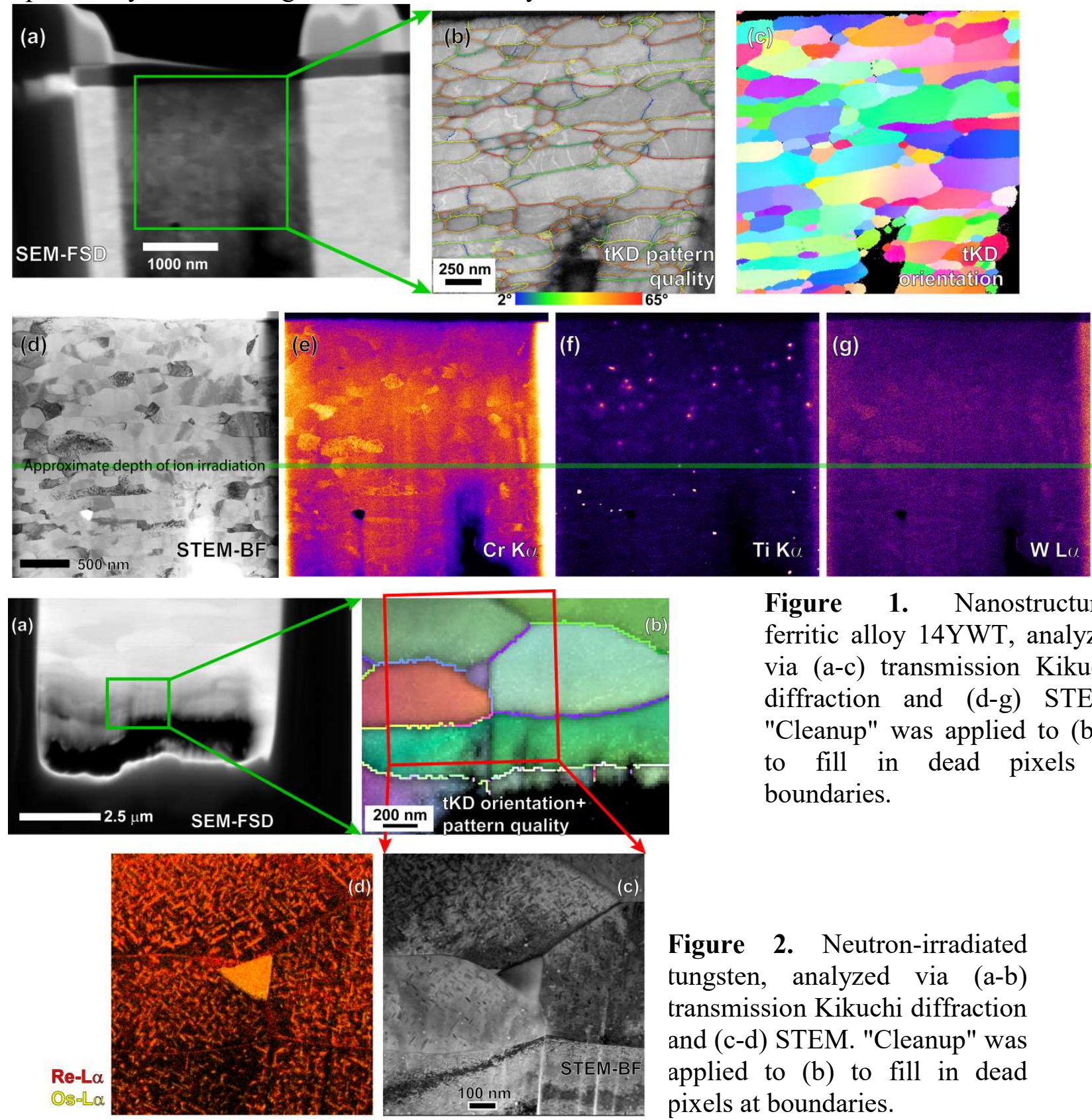

Figure 1. Nanostructured ferritic alloy 14YWT, analyzed via (a-c) transmission Kikuchi diffraction and (d-g) STEM. "Cleanup" was applied to (b-c) to fill in dead pixels at boundaries.

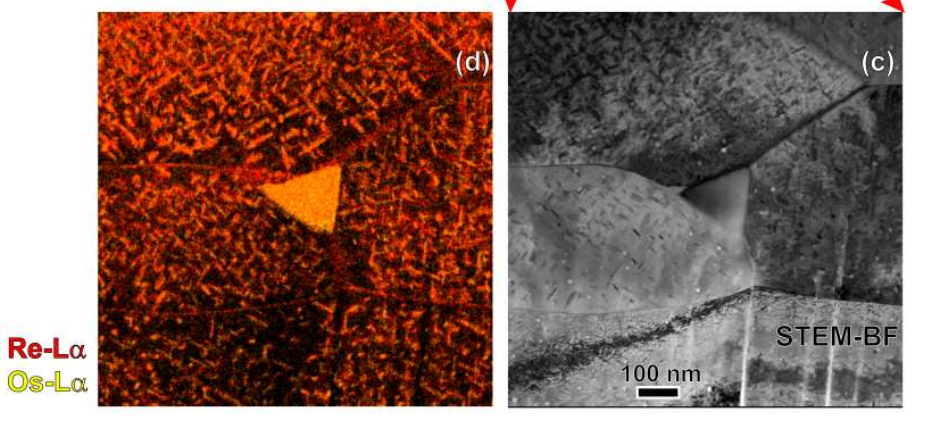

Figure 2. Neutron-irradiated tungsten, analyzed via (a-b) transmission Kikuchi diffraction and (c-d) STEM. "Cleanup" was applied to (b) to fill in dead pixels at boundaries. 